\title{
Cinco especies nuevas de Drosophila (Diptera, Drosophilidae) relacionadas con Araceae
}

\author{
Luz Marina Llangarí-Arizo (i) \& Violeta Rafael (i)
}

\begin{abstract}
Laboratorio de Genética Evolutiva, Escuela de Ciencias Biológicas, Pontificia Universidad Católica del Ecuador, Apartado: 17-01-2184, Quito, Ecuador. (luzmarinallangari@gmail.com; vrafael@puce.edu.ec)
\end{abstract}

ABSTRACT. Five new species of Drosophila (Diptera, Drosophilidae) related with Araceae. Drosophila anthurium sp. nov., Drosophila tsachila sp. nov., Drosophila guajalito sp. nov., Drosophila deloscolorados sp. nov. and Drosophila cartucho sp. nov. were captured in inflorescences of the family Araceae between 1800 and 2200 m of altitude in the Scientific Station Rio Guajalito, province of Santo Domingo de los Tsáchilas, Ecuador. The new species present surstylus and aedeagus elongated, and aedeagal apodeme very short. Analysis of external morphology and male terminalia indicate that the five new species do not belong to any of the Drosophila groups associated with flowers.

KEYWORDS. Anthurium, Cartucho, Colorados, Guajalito, Tsáchila.

RESUMEN. Drosophila anthurium sp. nov., Drosophila tsachila sp. nov., Drosophila guajalito sp. nov., Drosophila deloscolorados sp. nov. y Drosophila cartucho sp. nov. fueron capturadas en inflorescencias de la familia Araceae entre los 1800 y $2200 \mathrm{~m}$ de altitud en la Estación Científica Río Guajalito, provincia de Santo Domingo de los Tsáchilas, Ecuador. Las especies nuevas presentan surestilo y edeago largo, y apodema del edeago muy corto. Los análisis de la morfología externa y de la terminalia masculina indican que las cinco especies nuevas no pertenecen a ninguno de los grupos de Drosophila asociados a flores.

PALABRAS CLAVE. Anthurium, Cartucho, Colorados, Guajalito, Tsáchila.

Desde que STURTEvant (1921) encontró por primera vez a Drosophila florae y Drosophila lutzii en la corola de las flores tubulares de Datura (Solanaceae) y de Cucumis (Cucurbitaceae) respectivamente (BRNCIC, 1983), varias especies de Drosophila han sido observadas viviendo en una variedad de flores de diferentes familias.

BRNCIC (1983) mencionó que 140 especies de Drosophila estarían relacionadas con flores, y la mayoría de ellas son polífagas. Se han registrado algunos grupos del género Drosophila asociados con flores. Por ejemplo: el grupo Drosophila flavopilosa Frey, 1919 hasta el momento cuenta con 18 especies descritas estrechamente relacionadas con flores del género Cestrum (Solanaceae) (BRNCIC, 1966, 1983; RoBe et al., 2013; RAmos \& RAFAel, 2018). El grupo Drosophila bromeliae Patterson \& Stone, 1952, de origen neotropical, está integrado por 14 especies asociadas a flores de varias familias botánicas (VAL \& MARQUES, 1996; Silva \& MARTINS, 2004; GRIMALDI, 2016). Otro grupo asociado a flores de la familia Asteraceae es Drosophila onychophora Vilela \& Bächli, 1990, formado por 22 especies (HUNTER, 1979; Vilela \& BäChLI, 1990; HUnTER, 1992; Figuero \& RAfAel, 2011; Figuero et al., 2012). PIPKIN (1964) describió cuatro especies de Drosophila relacionadas con flores de los géneros Calathea y Heliconia (PIPKIN et al., 1966), y propuso el grupo natural Drosophila xanthopallescens Pipkin, 1964. VAZ et al. (2014) también reportan varias especies de Drosophila relacionadas a flores de Calathea y describen una nueva especie Drosophila calatheae que podría estar relacionada a los grupos $D$. xanthopallescens y $D$. bromeliae.

También se han registrado drosofilídeos en inflorescencias de Araceae. Las aráceas son plantas pequeñas terrestres, epífitas o acuáticas, muy largas, con cristales de oxalato de calcio, alcaloides, látex, canales mucilágenos; sin estípulas, inflorescencia indeterminada usualmente terminal; espádice sustentado por una bráctea (JARAMILLO \& GRIJALVA, 2010). En Ecuador, la familia Araceae comprende 21 géneros y 404 especies (JARAMILLO \& GRIJALVA, 2010) y su diversidad es mayor en los sitios más húmedos de elevaciones bajas y medias, y es menor en los sitios de tierras bajas secas o elevaciones muy altas (VARGAS et al., 2004).

En el trópico asiático, las inflorescencias de las aráceas Alocasia, Colocasia y Homalomena son sitios de reproducción específicos de moscas del género Colocasiomyia (Diptera, Drosophilidae) (CARSON \& OKADA, 1980; SHI et al., 2019). Por otro lado, en América Central se registró la relación entre algunas aráceas como Xanthosoma robustum y Drosophila 
aracea (HeED \& WheEler, 1957; TsaCAS \& Chassagnard, 1992), así como Monstera lentii y Drosophila monsterae (Vilela \& Prieto, 2018). En Ecuador, Llangarí \& Rafael (2017) detectaron a Drosophila sagitiffolli emergiendo de la inflorescencia de Xanthosoma sagitifolium.

En este trabajo se describen cinco especies nuevas del género Drosophila relacionadas a la familia botánica Araceae.

\section{MATERIALES Y MÉTODOS}

Área de estudio. Estación Científica Río Guajalito, Provincia de Santo Domingo de los Tsáchilas, Ecuador, (00 $13^{\prime} 48^{\prime \prime}$ S, $\left.78^{\circ} 49^{\prime} 16^{\prime \prime} \mathrm{W}\right)$. La estación comprende bosque maduro, bosque secundario y pastizales. En Río Guajalito, la segunda familia botánica de mayor riqueza después de Orchidaceae es Araceae, con 57 especies, de las cuales 46 son del género Anthurium (JaRAMillo \& GRIJALVA, 2010).

El muestreo se llevó a cabo desde los 1800 hasta los $2200 \mathrm{~m}$ de altitud. Las recolecciones de individuos se realizaron en los meses de septiembre 2010, marzo, junio, julio, agosto y septiembre 2011. Las drosófilas fueron capturadas directamente de las inflorescencias de Araceae con ayuda de un aspirador entomológico. También se recogieron inflorescencias, que posiblemente tenían huevos y larvas. Estas inflorescencias se conservaron en el laboratorio en frascos de vidrio con papel húmedo hasta la eclosión de los imagos. Con las hembras recolectadas se fundaron isolíneas y se mantuvieron en medio de cultivo de banano gelatina (RAFAEL et al., 2000)

La identificación de las especies se hizo mediante el análisis de la morfología externa, terminalia y la revisión de bibliografía especializada. Para el análisis de la terminalia se extrajo los dos últimos segmentos abdominales y se colocaron en microtubos con hidróxido de potasio $(\mathrm{KOH})$ al $10 \%$, posteriormente estos microtubos se hirvieron por 10 minutos. La terminalia de cada individuo se colocó en placas excavadas con glicerol. Para la descripción de las especies se utilizó la terminología propuesta por BäCHLI et al. (2004). Las especies fueron ilustradas usando un microscopio (Zeiss-46 70 86) con cámara lucida (Zeiss-47 4620 9900). Los dibujos se realizaron en el programa Adobe Illustrator ${ }^{\circledR}$. La toma de medidas para el cálculo de los índices se realizó con el programa AxioVision V4. Los valores entre paréntesis corresponden a las medidas de los paratipos.

El holotipo y paratipos se montaron en seco pegados en triángulos de cartulina, y sus terminalias se colocaron en un microtubo con glicerol. Todos los individuos fueron depositados en el Museo de Zoología, sección Invertebrados de la Pontificia Universidad Católica del Ecuador, Quito (QCAZ-I).

\section{Drosophila anthurium sp. nov.} (Figs 1-7, 35)

urn:lsid:zoobank.org:act:8991B243-0942482E-BBD8-7F5F81D6FF5C
Material tipo. Holotipo $\widehat{\widehat{\partial}}$ (disectado, montado en seco, terminalia en microtubo), Ecuador, Santo Domingo de los Tsáchilas, Estación Científica Río Guajalito $\left(00^{\circ} 13^{\prime} 48^{\prime}\right.$ 'S, 7849'16”'W, 1.800-2.200 msnm.), III.2011, L. M. Llangarí col., Llangarí \& Rafael det. (QCAZI 3099); Paratipos: $9 \hat{\jmath}$ (disectados, montados en seco, terminalia en microtubo), los mismos datos del holotipo, III.2011, VII.2011 (QCAZI 3100-3108).

Diagnosis. Tórax con una banda más clara en el centro, con seis hileras de pelos acrosticales entre las dorsocentrales anteriores. Cerdas escutelares basales divergentes. Cerda esternopleural media casi imperceptible. Cerci libres, prominentes, oscuros y microtricosos. Surestilo largo. Edeago quitinizado, largo y curvado, termina en dos láminas con el borde ventral aserrado y menos quitinizado que la parte dorsal.

Descripción del macho, holotipo y paratipos (individuos descritos y conservados en etanol). Morfología externa. Longitud total (cuerpo + alas) del holotipo $3.50 \mathrm{~mm}$. Longitud total (cuerpo + alas) de los paratipos 3.12-3.65. Color del cuerpo marrón amarillento.

Cabeza. Frente marrón. Longitud frontal 0.31 (0.30$0.34) \mathrm{mm}$. Índice frontal $1.30(1.20-1.31)$. Triángulo frontal amarillo. Triángulo ocelar marrón, cerca del 105(103-110)\% de la longitud, ocelos amarillos. Placa orbital amarilla; cerdas orbitales alineadas y equidistantes, distancia de or 3 a or1, $82(77-94) \%$ de or3 a vtm, radio or1/or3 $1.13(0.97-1.15)$, radio or2/or1 $0.39(0.40-0.58)$, cerda postocelar $41(35-47) \%$ y cerda ocelar 44 (39-43)\% de la longitud frontal, índice vt 0.77 (0.72-0.93). Frontal vitta amarilla. Gena y postgena marrón amarillento. Una cerda oral prominente. Carina marrón amarillenta, no prominente, no surcada. Proboscis amarilla. Ojos de color rojo vinoso, índice del ojo 1.18 (1.151.20). Segundo segmento antenal marrón amarillento; tercer segmento antenal amarillo. Arista plumosa con cinco ramas dorsales, dos ventrales, más la terminal bifurcada.

Tórax. Marrón amarillento con una banda clara en el centro, longitud 1.10 (1.11-1.17) mm. Índice h 1.29 (0.98-1.40). Seis hileras de pelos acrosticales entre las cerdas dorsocentrales anteriores. Distancia transversal de las cerdas dorsocentrales 151 (151-191)\% de la distancia longitudinal, índice dc 0.58 (0.58-0.61). Escutelo del mismo color que el tórax, distancia entre las cerdas escutelares apicales 62 (56-90)\%
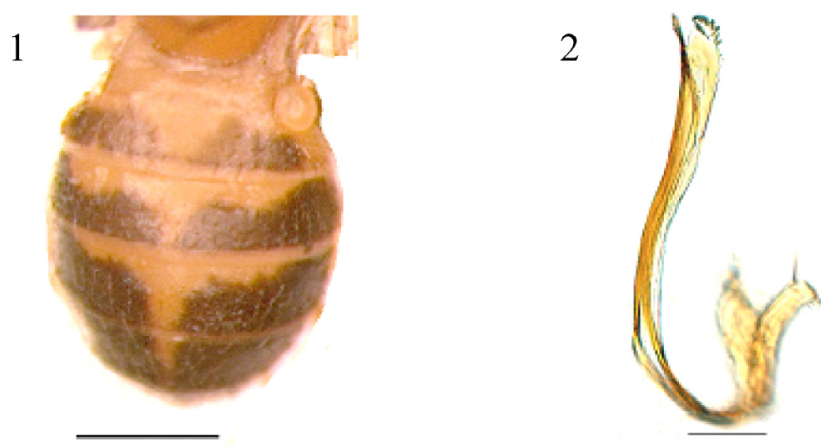

Figs 1, 2. Drosophila anthurium sp. nov., paratipos $\widehat{\partial}: 1$, abdomen, vista dorsal (Escala: $0.5 \mathrm{~mm}$ ); 2, edeago vista lateral (Escala: $0.1 \mathrm{~mm}$ ). 
de la distancia entre las cerdas apicales y basales. Cerdas escutelares basales divergentes, índice escutelar 1.40 (1.331.46). Cerda esternopleural media casi imperceptible, índice esterno 0.43 (0.39-0.47). Patas amarillas.

Alas amarillas. Longitud del ala 2.70 (2.50-2.75) mm. Índices alares 2.50 (2.48-2.53); $\mathrm{C}=4.40$ (4.38-4.47), $\mathrm{ac}=1.60(1.54-1.68), \mathrm{hb}=0.30(0.25-0.38), 4 \mathrm{c}=0.60(0.55-0.63)$, $4 \mathrm{v}=1.60(1.55-1.64), 5 \mathrm{x}=1.30(1.27-1.35), \mathrm{M}=0.40(0.39-$ $0.42)$ y prox. $x=0.50(0.47-0.51)$.
Abdomen amarillo. Primer tergito amarillo; con línea media dorsal del segundo al quinto tergito. Segundo tergito pigmentado las $3 / 4$ partes desde el borde posterior, los lados sin pigmentación; tercer tergito con pigmentación que ocupa casi todo el ancho del tergito y se extiende hacia los lados; cuarto tergito pigmentado las $3 / 4$ partes y se extiende hacia los lados; quinto tergito con un área clara en la parte media, y sexto tergito totalmente pigmentado (Fig. 1).
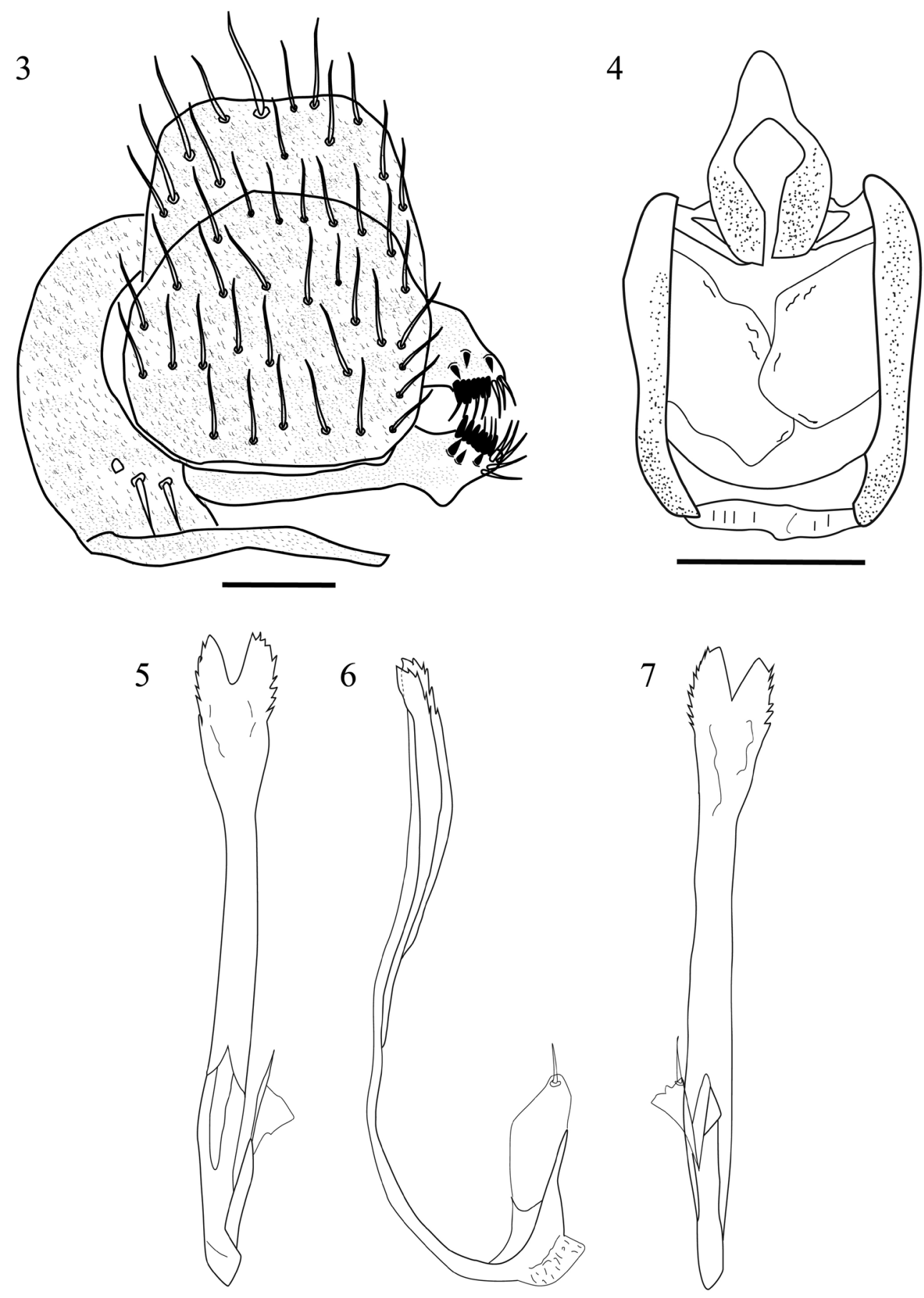

Figs 3-7. Drosophila anthurium sp. nov., holotipo đ̊: 3, epandrio; 4, hipandrio y gonopodio; 5-7, edeago y paráfisis, vistas dorsal, lateral y ventral, respectivamente (Escala: $0.1 \mathrm{~mm}$ ). 
Terminalia externa. Cerci libres, prominentes, oscuros, microtricosos y con muchas cerdas. Epandrio microtricoso, con tres cerdas en la parte lateral, lóbulo ventral del epandrio delgado, largo, muy quitinizado y desnudo. Surestilo modificado largo, quitinizado y nace cerca de la parte dorsal del epandrio; surestilo derecho e izquierdo con siete dientes primarios (7-8), tres dientes secundarios puntiagudos y siete cerdas marginales (6-7) (Fig. 3).

Terminalia interna. Hipandrio en forma de escudo con el contorno muy quitinizado, gonopodio poco quitinizado, con arco dorsal sostenido por dos formaciones quitinosas (Fig. 4). Edeago quitinizado, largo, curvado, termina en dos láminas con el borde ventral aserrado y menos quitinizado que la parte dorsal. Apodema del edeago casi inexistente, rama ventral alargada y delgada, paráfisis ovalada con una cerda en el extremo distal (Figs 2, 5-7).

Ecología. Esta especie fue capturada en las inflorescencias de Anthurium sp. (Fig. 35), además en el laboratorio emergió un individuo de la inflorescencia de Anthurium sp. colectada en el campo.

Etimología. El nombre Drosophila anthurium sp. nov. hace referencia al género botánico hospedero.

\section{Drosophila tsachila sp. nov.}

(Figs 8-14, 36)

urn:1sid:zoobank.org:act:21D554C6-5BC8-4070-

B855-2F9780F8AFB7

Material tipo. Holotipo $\widehat{\partial}$ (disectado, montado en seco, terminalia en microtubo), Ecuador, Santo Domingo de los Tsáchilas, Estación Científica Río Guajalito ( $00^{\circ} 13^{\prime} 48^{\prime \prime} \mathrm{S}$, 7849’16”W, 1.800-2.200 msnm.), VII.2011, L. M. Llangarí col., Llangarí \& Rafael det. (QCAZI 3079); Paratipos: 90 (disectados, montados en seco, terminalia en microtubo), los mismos datos del holotipo, III.2011, VII.2011 (QCAZI 3080-3088).

Diagnosis. Tórax con seis hileras de pelos acrosticales entre las dorsocentrales anteriores. Cerdas escutelares basales divergentes. Cerda esternopleural media casi imperceptible. Cerci libres, prominentes, y microtricosos. Surestilo muy largo. Edeago quitinizado, largo y curvo, los extremos ventral y dorsal ligeramente quitinizados, y unidos por una membrana con microproyecciones y una hendidura en vista dorsal.

Descripción del macho, holotipo y paratipos (individuos descritos y conservados en etanol). Morfología externa. Longitud total (cuerpo + alas) del holotipo $3.60 \mathrm{~mm}$. Longitud total (cuerpo + alas) de los paratipos 3.55-3.83 $\mathrm{mm}$. Color del cuerpo marrón amarillento.

Cabeza. Frente marrón. Longitud frontal 0.27 (0.28$0.33) \mathrm{mm}$. Índice frontal 1.08 (1.20-1.50). Triángulo frontal marrón amarillento. Triángulo ocelar marrón, cerca del 33(34$39) \%$ de la longitud, ocelos amarillos. Placa orbital amarilla, cerda orbital media cerca a la cerda orbital anterior y hacia el borde externo de la placa orbital, distancia de or3 a or1 $84(90-92) \%$ de or3 a vtm, radio or1/or3 $0.76(0.86-0.88)$, radio or2/or1 $0.54(0.39-0.50)$, cerda postocelar $46(47-50) \%$ y cerda ocelar $71(82-85) \%$ de la longitud frontal; índice vt
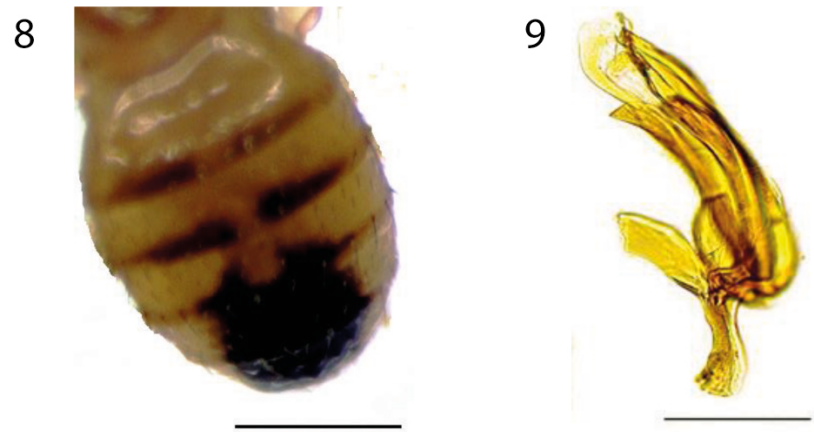

Figs 8, 9. Drosophila tsachila sp. nov., paratipos $\delta^{\wedge}: 8$, abdomen, vista dorsal (Escala: $0.5 \mathrm{~mm}$ ); 9, edeago vista lateral (Escala: $0.1 \mathrm{~mm}$ ).

0.88 (0.92-1.04). Frontal vitta marrón amarillento. Gena y postgena marrón amarillento. Una cerda oral prominente. Carina amarilla, no prominente, no surcada. Proboscis amarilla. Ojos de color rojo vinoso, índice del ojo 1.13 (1.181.50). Segundo y tercer segmento antenal marrón. Arista plumosa con cinco ramas dorsales, dos ventrales más la terminal bifurcada.

Tórax marrón amarillento, longitud 1.05 (0.981.10) $\mathrm{mm}$. Índice h 0.67 (0.68-0.79). Seis hileras de pelos acrosticales entre las dorsocentrales anteriores. Distancia transversal de las cerdas dorsocentrales $40(41-43) \%$ de la distancia longitudinal, índice dc 0.58 (0.64-0.66). Escutelo del mismo color que el tórax, distancia entre las cerdas escutelares apicales $142(106-133) \%$ de la distancia entre las cerdas apicales y basales. Cerdas escutelares basales divergentes, índice escutelar 0.49 (0.66-0.70). Cerda esternopleural media casi imperceptible, índice esterno 0.80 (0.63-0.93). Patas amarillas.

Alas amarillas. Longitud del ala $2.20 \mathrm{~mm}$ (2.31-2.43) mm. Índices alares: alar=2.10 (2.43-2.75); $\mathrm{C}=3.50$ (3.54-3.73); $\mathrm{ac}=1.90$ (2.09-2.25); $\mathrm{hb}=0.40(0.27-0.33)$; $4 \mathrm{c}=0.80(0.72-0.73) ; 4 \mathrm{v}=1.90(1.68-1.77) ; 5 \mathrm{x}=1.20(1.15-$ $1.52) ; \mathrm{M}=0.50(0.37-0.48)$ y prox. $\mathrm{x}=0.70(0.55-.0 .70)$.

Abdomen amarillo. Primer tergito amarillo; con línea media dorsal del segundo al cuarto tergito. Segundo tergito con pigmentación tenue en el borde posterior; tercer y cuarto tergito con pigmentación en forma de triángulo en el borde posterior; quinto y sexto tergito presentan una mancha oscura en la parte dorsal del tergito. Los lados de todos los tergitos amarillos (Fig. 8).

Terminalia externa. Cerci libres, prominentes, microtricosos y con cerdas. Parte dorsal del epandrio microtricoso, sin cerdas. Lóbulo ventral del epandrio delgado y desnudo. Surestilo muy largo y quitinizado. Surestilo derecho e izquierdo con siete dientes primarios, cuatro (3-4) dientes secundarios quitinizados y puntiagudos y seis (6-7) cerdas marginales (Fig. 10).

Terminalia interna. Hipandrio muy quitinizado en forma de escudo, con arco dorsal, gonopodios rectangulares (Fig. 11). Edeago quitinizado, largo y curvo, los extremos ventral y dorsal ligeramente quitinizados, y unidos por una membrana con microproyecciones y una hendidura en vista dorsal. Apodema del edeago corto, ancho y membranoso, 
rama ventral corta y delgada, paráfisis rectangular con una cerda en la parte distal (Figs 9, 12-14).

Ecología. Individuos capturados sobre las inflorescencias de Araceae 1 (Fig. 36).

Etimología. El nombre de esta especie se refiere al grupo étnico de los Tsáchilas que viven en las estribaciones occidentales de los Andes en la provincia de Santo Domingo de los Tsáchilas, lugar de colecta. En lengua tsáfiki, hablada por la etnia de los Tsáchilas, Tsa, significa verdadero y chila, significa gente.

10

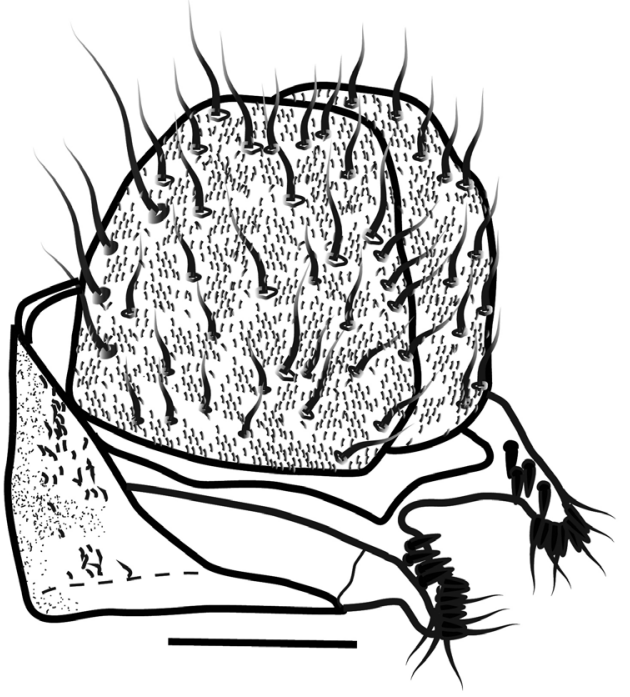

\section{Drosophila guajalito sp. nov.} (Figs 15-21)

urn:1sid:zoobank.org:act:753A12A5-DC4343CE-9A75-72DA2520736E

Material tipo. Holotipo $\widehat{\partial}$ (disectado, montado en seco, terminalia en microtubo), Ecuador, Santo Domingo de los Tsáchilas, Estación Científica Río Guajalito (00¹3'48”S, 7849'16"W, 1.800-2.200 msnm.), VII.2011, L. M. Llangarí col., Llangarí \& Rafael det. (QCAZI 3109); Paratipos: $9 ð$

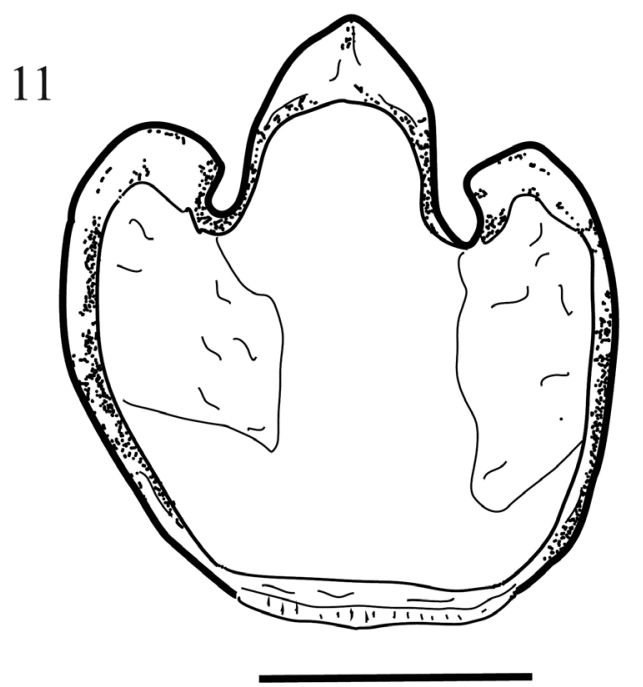

12
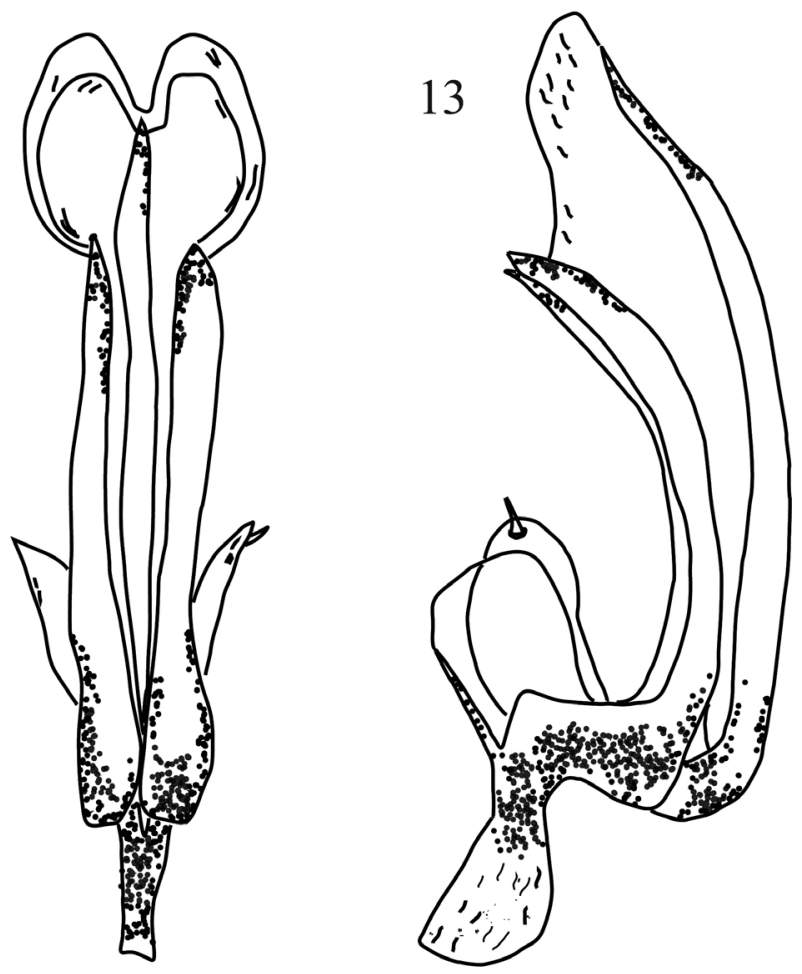

14

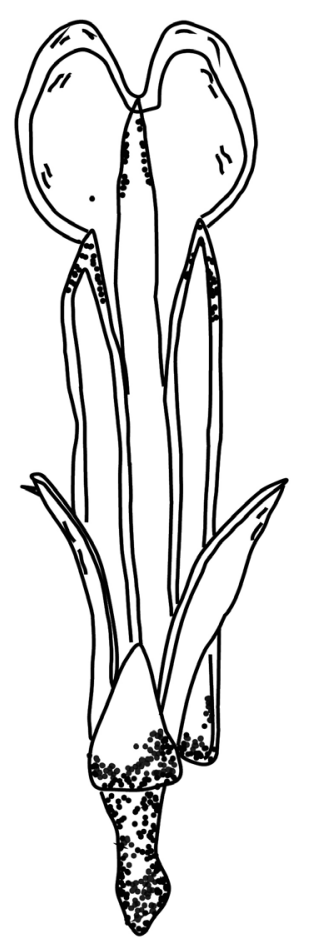

Figs 10-14. Drosophila tsachila sp. nov., holotipo ổ: 10, epandrio; 11, hipandrio y gonopodio; 12-14, edeago y paráfisis, vistas dorsal, lateral y ventral, respectivamente (Escala: $0.1 \mathrm{~mm}$ ). 
(disectados, montados en seco, terminalia en microtubo), los mismos datos del holotipo, VII.2011, IX.2011 (QCAZI 3110-3118).

Diagnosis. Tórax con seis hileras de pelos acrosticales entre las dorsocentrales anteriores. Cerdas escutelares basales divergentes. Cerda esternopleural media ausente. Cerci libres, prominente quitinizada con muchas cerdas. Surestilo largo y delgado. Edeago largo, curvado con dos ramas: una rama corta quitinizada que termina en dos puntas; una rama larga que se ensancha.

Descripción del macho, holotipo y paratipos (individuos descritos y conservados en etanol). Morfología externa. Longitud total (cuerpo + alas) del holotipo $3.30 \mathrm{~mm}$. Longitud total (cuerpo + alas) de los paratipos 2.98-3.40. Color del cuerpo marrón amarillento.

Cabeza. Frente marrón. Longitud frontal $0.28(0.30-$ 0.32). Índice frontal $0.80(0.83-1.00)$. Triángulo frontal amarillo; triángulo ocelar marrón amarillento, cerca del $36(34-45) \%$ de la longitud, ocelos amarillos. Placa orbital marrón, cerda orbital media cerca a la cerda orbital posterior (en algunos individuos la orbital media es equidistante de la cerda anterior y de la cerda posterior) y hacia al borde externo de la placa orbital. Distancia de or3 a or1 $1.00(1.00-1.17)$ de or3 a vtm, radio or1/or3 1.00 (0.90-1.12), radio or2/or1 $0.30(0.28-0.34)$, cerda postocelar $43(38-44) \%$ y cerda ocelar $61(60-63) \%$ de la longitud frontal; índice vt 1.00 (0.98-1.05). Frontal vitta marrón amarillento. Gena y postgena marrón amarillento. Una cerda oral prominente. Carina marrón amarillenta, no prominente, no surcada. Proboscis amarilla. Ojos de color rojo vinoso, índice del ojo 1.08 (1.12-1.46). Segundo segmento de las antenas marrón; tercer segmento, blanco polinoso. Arista plumosa con cuatro ramas dorsales y dos ventrales, más la terminal bifurcada.

Tórax. Marrón amarillento, longitud 0.93 (0.87$0.97) \mathrm{mm}$. Índice h 0.94 (0.82-0.91). Seis hileras de pelos acrosticales entre las cerdas dorsocentrales anteriores. Distancia transversal de las cerdas dorsocentrales 32(33$35) \%$ de la distancia longitudinal, índice de 0.59 (0.56-0.60). Escutelo del mismo color que el tórax, distancia entre las cerdas escutelares apicales 125(125-129)\% de la distancia entre las cerdas apicales y basales. Cerdas escutelares basales divergentes, índice escutelar 1.03 (0.97-1.00). Cerda esternopleural media ausente (considerando 20 individuos analisados), índice esterno 0.59 (0.60-0.62). Patas amarillas.

Alas amarillas. Longitud del ala $2.20 \mathrm{~mm}$. Índices alares: alar $=1.90(2.07-2.28) ; \mathrm{C}=3.20(4.24-4.28)$; $\mathrm{ac}=1.60(1.59-1.68) ; \mathrm{hb}=0.30(0.35-0.37) ; 4 \mathrm{c}=0.80(0.60-$ $0.62) ; 4 \mathrm{v}=1.80(1.66-1.80) ; 5 \mathrm{x}=1.05(1.01-1.23) ; \mathrm{M}=$ $0.41(0.44-0.50)$ y prox. $x=0.60(0.61-0.65)$.

Abdomen. Marrón amarillento. Primer tergito amarillo; segundo tergito con dos triángulos oscuros a cada lado de la línea media dorsal; tercer tergito con una franja oscura en el borde posterior y con una mancha oscura triangular en el centro del tergito; cuarto tergito marrón ligeramente más claro en el borde anterior; quinto y sexto tergito marrón (Fig. 15).
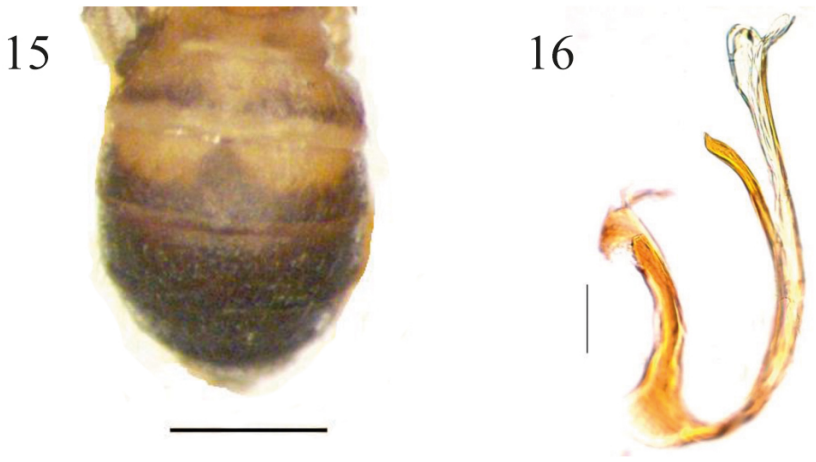

Figs 15,16. Drosophila guajalito sp. nov., paratipos $\precsim$ : 15 , abdomen, vista dorsal (Escala: $0.5 \mathrm{~mm}$ ); 16, edeago vista lateral (Escala: $0.1 \mathrm{~mm}$ ).

Terminalia externa. Cerci libres, prominentes quitinizados con muchas cerdas y pelos. Epandrio muy quitinizado con finos pelos sin cerdas. Lóbulo ventral del epandrio largo y desnudo. Surestilo largo y delgado; surestilo derecho con diez (9-10) dientes primarios, cuatro (4-6) dientes secundarios y siete (7-8) cerdas marginales; surestilo izquierdo con diez (9-10) dientes primarios, cinco (4-6) dientes secundarios y siete (7-8) cerdas marginales (Fig. 17).

Terminalia interna. Hipandrio muy quitinizado en forma de escudo, con arco dorsal quitinizado, gonopodios menos quitinizados con una cerda (Fig.18). Edeago quitinizado, largo, curvado con dos ramas: una rama corta (rc) quitinizada que termina en dos puntas; otra rama larga (rl) que se ensancha, menos quitinizada, que termina en dos valvas y en la parte dorsal presenta dos proyecciones laminares. Apodema del edeago casi inexistente, rama ventral quitinizada larga (Figs 16, 19-21).

Ecología. Individuos capturados en inflorescencias de Xanthosoma sagittifolium y otras aráceas.

Etimología. El nombre de esta especie se refiere al lugar de colecta, el Bosque Río Guajalito, ubicado en la Provincia de Santo Domingo de los Tsáchilas.

\section{Drosophila deloscolorados sp. nov. (Figs 22-28, 35-37) urn:1sid:zoobank.org:act:AD9FA1DF-29DE- 4505-B1C2-16897D01C996}

Material tipo. Holotipo $\widehat{\delta}$ (disectado, montado en seco, terminalia en microtubo), Ecuador, Santo Domingo de los Tsáchilas, Estación Científica Río Guajalito ( $00^{\circ} 13$ '48”S, 7849’16”W, 1.800-2.200 msnm.), III.2011, L.M. Llangarí col., Llangarí \& Rafael det. (QCAZI 3089); Paratipos: 9 $\widehat{ }$ (disectados, montados en seco, terminalia en microtubo), los mismos datos del holotipo, III.2011, VII.2011 (QCAZI 3090-3098).

Diagnosis. Tórax con seis hileras de pelos acrosticales entre las dorsocentrales anteriores, cerdas escutelares basales divergentes. Cerda esternopleural media es $1 / 5$ de la cerda esternopleural anterior. Edeago quitinizado, largo, curvado, parte distal ensanchada y aserrada, paráfisis larga que termina en dos proyecciones a modo de cuerno. 

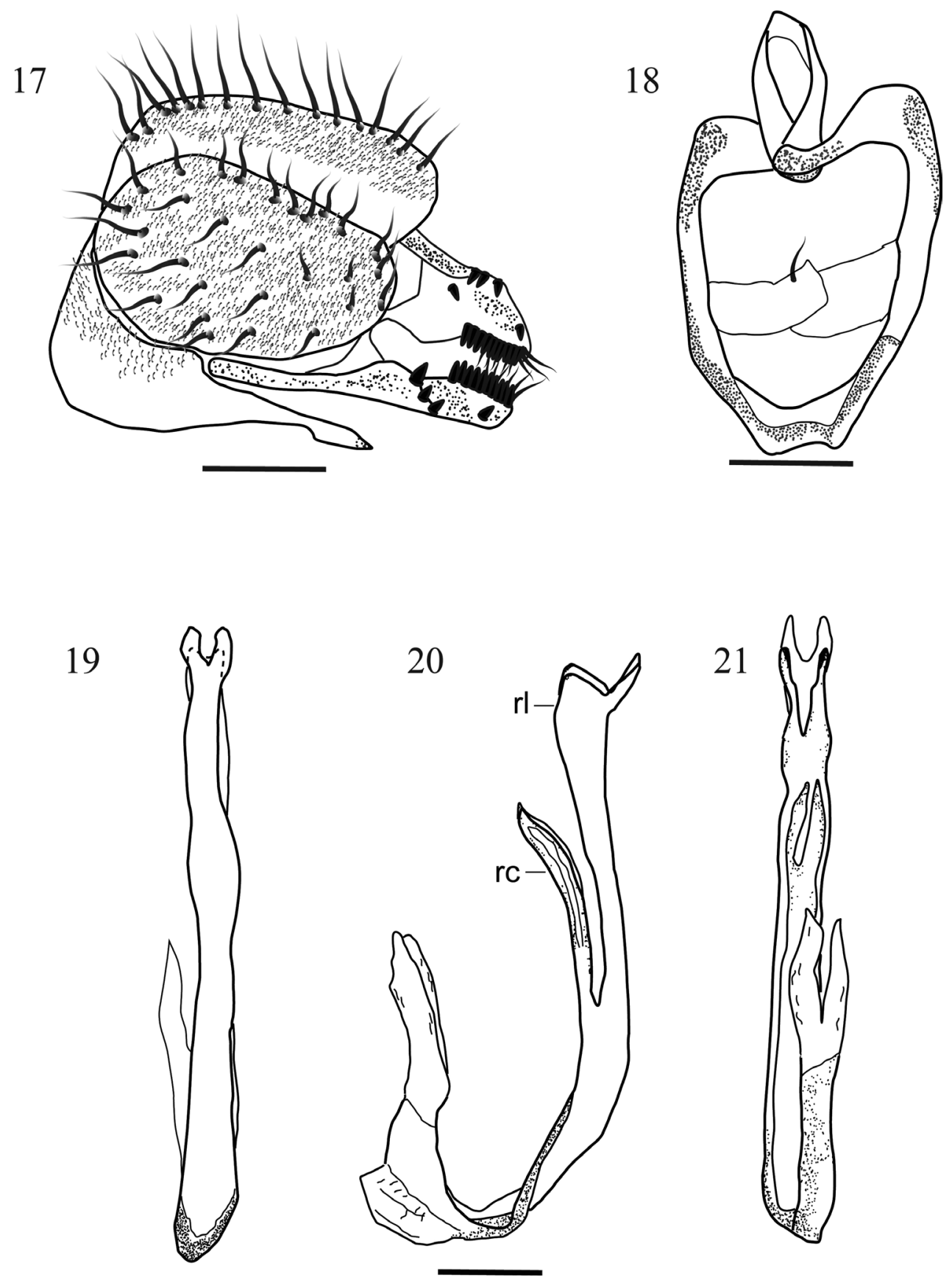

Figs 17-21. Drosophila guajalito sp. nov., holotipo ổ: 17, epandrio; 18, hipandrio y gonopodio; 19-21, edeago y paráfisis, vistas dorsal, lateral y ventral, respectivamente ( $\mathrm{rl}$, rama larga; rc, rama corta) (Escala: $0.1 \mathrm{~mm}$ ).

22

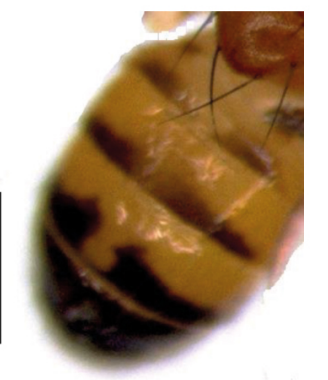

23

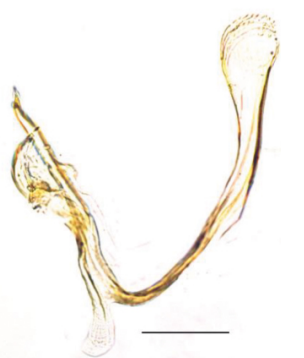

Figs 22, 23. Drosophila deloscolorados sp. nov., paratipos $\partial^{\lambda}: 22$, abdomen, vista dorsal (Escala: $0.5 \mathrm{~mm}$ ); 23, edeago, vista lateral (Escala: $0.1 \mathrm{~mm}$ ).
Descripción del macho, holotipo y paratipos (individuos descritos y conservados en etanol). Morfología externa. Longitud total (cuerpo + alas) del holotipo $3.50 \mathrm{~mm}$. Longitud total (cuerpo + alas) de los paratipos 3.26-3.70 $\mathrm{mm}$. Color del cuerpo amarillo.

Cabeza. Frente marrón. Longitud frontal 0.33 (0.28$0.31) \mathrm{mm}$. Índice frontal $0.97(0.93-0.96)$. Triángulo frontal marrón amarillento. Triángulo ocelar marrón amarillento, cerca del 34(33-36)\% de la longitud, ocelos amarillos. Placa orbital amarilla; cerda orbital media ubicada más cerca de la cerda orbital anterior y ligeramente hacia el exterior 
de la placa orbital. Distancia de or3 a or1 $85(83-85) \%$ de or3 a vtm, radio or1/or3 $0.82(0.78-0.80)$, radio or $2 /$ or 1 $0.22(0.17-0.21)$, cerda postocelar $31(40-46) \%$ y cerda ocelar $64(65-67) \%$ de la longitud frontal; índice vt 0.92 (0.78-0.80). Frontal vitta amarilla. Gena y postgena amarillas. Una cerda oral prominente. Carina amarilla, ligeramente prominente, no surcada. Proboscis amarilla. Ojos de color rojo vinoso, índice del ojo 1.37 (1.05-1.58). Segundo segmento antenal marrón amarillento; tercer segmento blanco polinoso. Arista marrón plumosa, con tres ramas dorsales y dos ventrales, más la terminal bifurcada.
Tórax. Amarillo, longitud 1.20 (1.10-1.45) mm. Índice h 0.80 (1.00-1.20). Seis hileras de pelos acrosticales entre las cerdas dorsocentrales anteriores. Distancia transversal de las cerdas dorsocentrales $56(51-53) \%$ de la distancia longitudinal, índice de 0.62 (0.51-0.58). Escutelo del mismo color que el tórax, distancia entre las cerdas escutelares apicales $158(146-154) \%$ de la distancia entre las cerdas apicales y basales. Cerdas escutelares basales divergentes, índice escutelar 1.00 (0.98-1.02). Cerda esternopleural media es $1 / 5$ de la cerda esternopleural anterior, índice esterno $0.32(0.33-0.35)$. Patas amarillas.

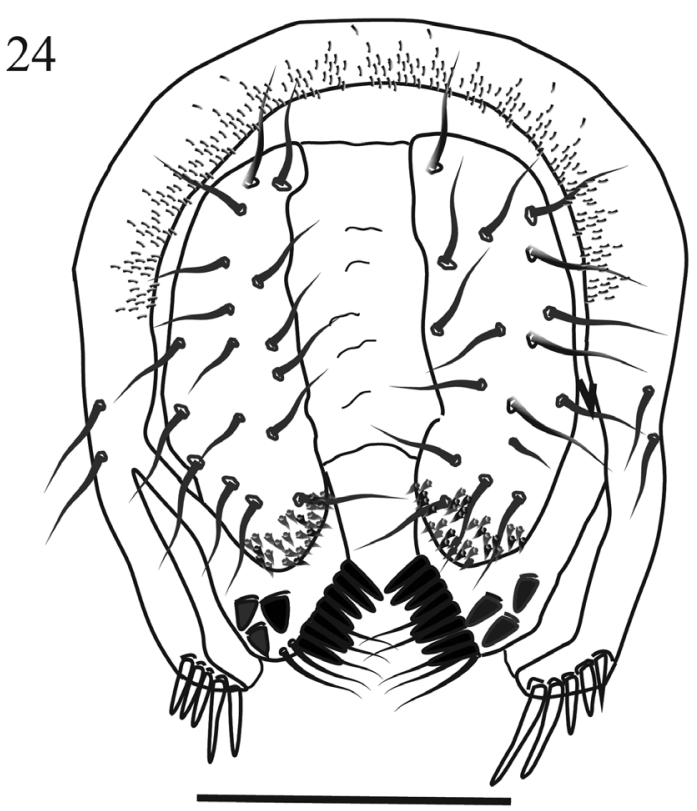

26

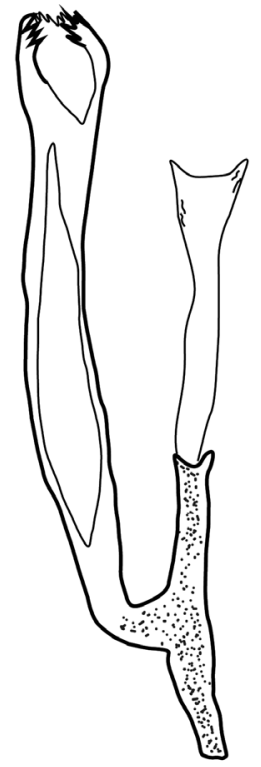

27

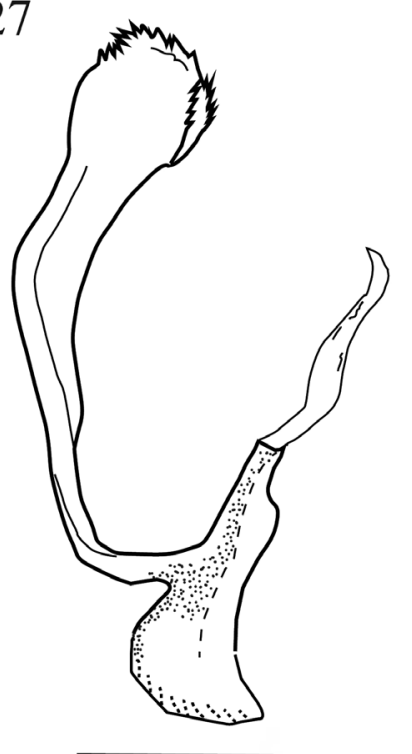

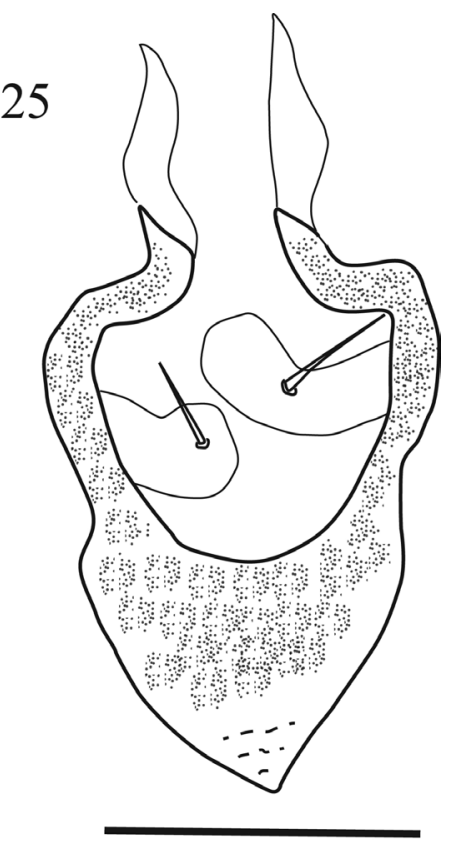

28

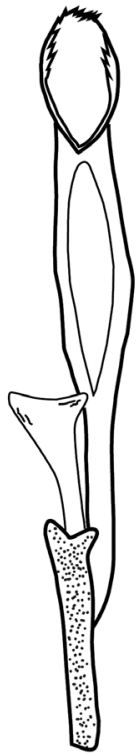

Figs 24-28. Drosophila deloscolorados sp. nov., holotipo ổ: 24, epandrio; 25, hipandrio y gonopodio; 26-28, edeago y paráfisis, vistas dorsal, lateral y ventral, respectivamente (Escala: $0.1 \mathrm{~mm}$ ). 
Alas amarillas. Longitud del ala $2.68(2.20-2.71) \mathrm{mm}$. Índices alares: $\mathrm{alar}=2.62(2.32-2.65) ; \mathrm{C}=5.23(4.61-5.21)$; $\mathrm{ac}=1.65(1.54-1.55), \mathrm{hb}=0.26(0.30-0.35) ; 4 \mathrm{c}=0.54(0.51-0.62)$ ; $4 \mathrm{v}=1.79(1.73-1.76) ; 5 \mathrm{x}=1.17(1.22-1.25) ; \mathrm{M}=0.38(0.38$ $0.46)$ y prox. $\mathrm{x}=0.52(0.50-0.60)$.

Abdomen amarillo, con línea media dorsal del primero al cuarto tergito. Primer tergito amarillo con dos manchas oscuras en el borde posterior; segundo tergito con una franja oscura en el borde posterior que se adelgaza hacia los lados; tercer tergito con una franja oscura en el borde posterior que alcanza casi la mitad del tergito; cuarto tergito con una franja oscura en el borde posterior y con una hendidura pronunciada en el centro; quinto y sexto tergito marrón (Fig. 22).

Terminalia externa. Cerci libres, microtricosos sólo en la parte inferior. Epandrio microtricoso sin cerdas en la parte dorsal, con dos cerdas en la parte lateral y en la parte baja con dos cerdas largas y tres cerdas pequeñas gruesas. Surestilo rectangular; surestilo derecho con siete (7-8) dientes primarios, tres (3-4) dientes secundarios y cinco cerdas marginales; surestilo izquierdo con ocho (78) dientes primarios, tres (3-4) dientes secundarios y cinco cerdas marginales (Fig. 24).

Terminalia interna. Hipandrio ovalado con el contorno quitinizado sin arco dorsal, margen anterior membranoso, gonopodio membranoso de forma ovalada con una cerda larga (Fig. 25). Edeago quitinizado, largo, curvado, parte distal ensanchada y aserrada, paráfisis larga, termina en dos proyecciones pequeñas a modo de cuerno, rama ventral desarrollada, apodema del edeago corto y membranoso (Figs 23, 26-28).

Ecología. Individuos capturados en las inflorescencias de Anthurium spp. (Fig. 35), Araceae 1 (Fig. 36), Araceae 2 (Fig. 37).

Etimología. El nombre de los colorados es un adjetivo con el que se conoce a los integrantes de la etnia de los Tsáchilas, porque se pintan el rostro y la cabeza con semillas de achiote (Bixa orellana L.). Los colorados habitan en la provincia de Santo Domingo de los Tsáchilas, lugar de colecta.

\section{Drosophila cartucho sp. nov.}

(Figs 29-34)

urn:lsid:zoobank.org:act:8CE950AC-20E2-461DA30F-B71D6791C91E
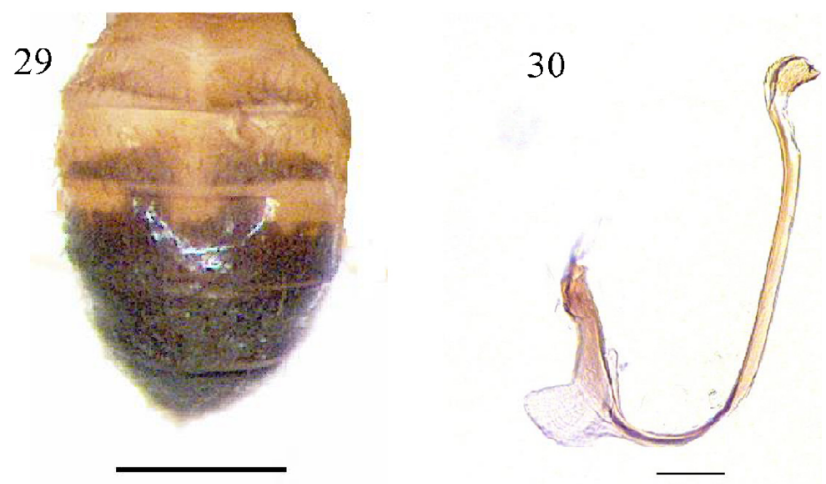

Figs 29, 30. Drosophila cartucho sp. nov., paratipos ${ }^{\wedge}: 29$, abdomen, vista dorsal (Escala: $0.5 \mathrm{~mm}$ ); 30, edeago, vista lateral (Escala: $0.1 \mathrm{~mm}$ ).
Material tipo. Holotipo $\widehat{\widehat{\gamma}}$ (disectado, montado en seco, terminalia en microtubo), Ecuador, Santo Domingo de los Tsáchilas, Estación Científica Río Guajalito ( $00^{\circ} 13$ '48”S, 7849'16”W, 1.800-1.890 msnm.), III.2011, L. M. Llangarí col., Llangarí \& Rafael det. (QCAZI 3119); Paratipos: $9{ }^{\lambda}$ (disectados, montados en seco, terminalia en microtubo), los mismos datos del holotipo, III.2011, VII.2011 (QCAZI 3120-3128).

Diagnosis. Tórax con seis hileras de pelos acrosticales entre las dorsocentrales anteriores. Cerdas escutelares basales divergentes. Cerda esternopleural media $1 / 5$ de la anterior. Edeago quitinizado, largo en forma de "C", el extremo distal ensanchado y borde ventral aserrado.

Descripción del macho, holotipo y paratipos (individuos descritos y conservados en etanol). Morfología externa. Longitud total (cuerpo + alas) del holotipo $3.20 \mathrm{~mm}$. Longitud total (cuerpo + alas) de los paratipos 3.05-3.32 $\mathrm{mm}$. Color del cuerpo amarillo.

Cabeza. Frente marrón. Longitud frontal 0.28 (0.250.28). Índice frontal 1.17 (0.96-1.16). Triángulo frontal amarillo, triángulo ocelar marrón amarillento, cerca del $39(38-42) \%$ de la longitud, ocelos amarillos. Placa orbital amarilla, cerda orbital media cerca a la cerda orbital anterior y cerca del borde externo de la placa orbital, distancia de or3 a or $183(83-86) \%$ de or3 a vtm, radio or $1 /$ or3 $0.95(0.95-0.97)$, radio or 2 or 10.38 (0.37-0.41), cerda postocelar 54(52-54)\% y cerda ocelar $72(69-73) \%$ de la longitud frontal; índice vt 1.04 (1.00-1.07). Frontal vitta amarilla. Gena y postgena amarillas. Una cerda oral prominente. Carina amarilla, no prominente, no surcada. Proboscis amarilla. Ojos de color rojo vinoso, índice del ojo 1.13 (1.12-1.20). Segundo segmento antenal amarillo; tercer segmento blanco polinoso. Arista plumosa con cuatro ramas dorsales y dos ventrales, más la terminal bifurcada.

Tórax marrón amarillento, longitud 0.91 (0.89$0.91) \mathrm{mm}$. Índice h 0.88 (0.88-0.93). Seis hileras de pelos acrosticales entre las cerdas dorsocentrales anteriores. Distancia transversal de las cerdas dorsocentrales 45(49$53) \%$ de la distancia longitudinal, índice de 0.55 (0.55-0.59). Escutelo marrón amarillento ligeramente más oscuro en el borde, distancia entre las cerdas escutelares apicales 130(127$130) \%$ de la distancia entre las cerdas apicales y basales. Cerdas escutelares anteriores divergentes, índice escutelar 0.95 (0.91-0.94). Cerda esternopleural media es $1 / 5$ de la cerda esternopleural anterior, índice esterno 0.64 (0.61-0.65). Patas amarillas.

Alas amarillas. Longitud del ala $2.44(1.86-2.44) \mathrm{mm}$. Índices alares: alar $=2.05(2.00-2.30) ; \mathrm{C}=3.80(3.70-4.02)$; $\mathrm{ac}=1.72(1.61-1.70) ; \mathrm{hb}=0.46(0.31-0.44) ; 4 \mathrm{c}=0.60(0.58-$ $0.64) ; 4 \mathrm{v}=1.50(1.50-1.71) ; 5 \mathrm{x}=1.20(1.12-1.25) ; \mathrm{M}=$ $0.40(0.38-0.42)$ y prox. $x=0.50(0.50-0.53)$.

Abdomen marrón amarillento. Primer tergito amarillo; segundo tergito amarillo con el borde posterior ligeramente marrón que se adelgaza a los lados hasta desaparecer; tercer tergito con áreas claras en la parte anterior y una franja 
oscura a lo largo del tergito que se adelgaza a los lados hasta desaparecer; cuarto tergito oscuro en sus 2/3 partes. En los lados de la línea media dorsal la pigmentación alcanza el borde anterior del tergito y forma un área clara; quinto y sexto tergito oscuro a todo lo ancho y largo (Fig. 29).

Terminalia externa. Cerci libres. La parte dorsal del epandrio microtricoso con cuatro cerdas, la parte lateral media con tres cerdas y el lóbulo ventral del epandrio con cinco (5-6) cerdas. Surestilo de forma rectangular sin microproyecciones; surestilo derecho e izquierdo con siete
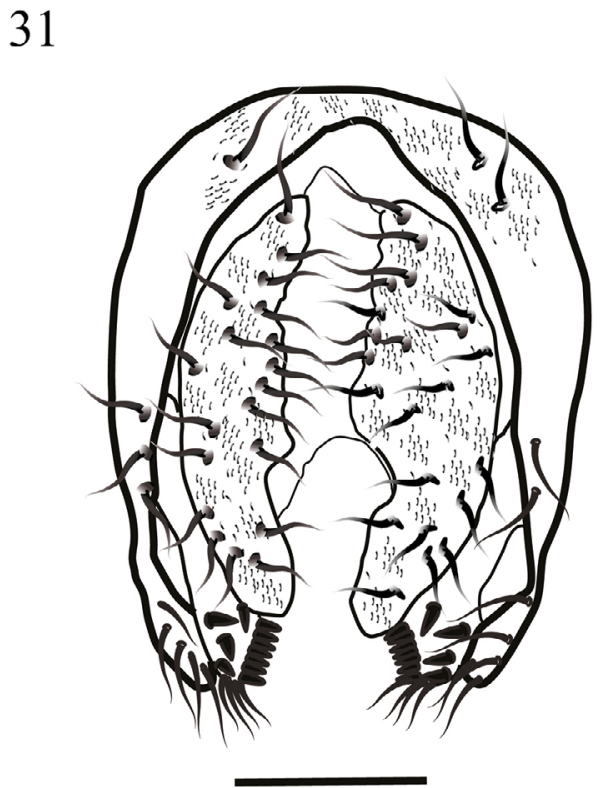

33

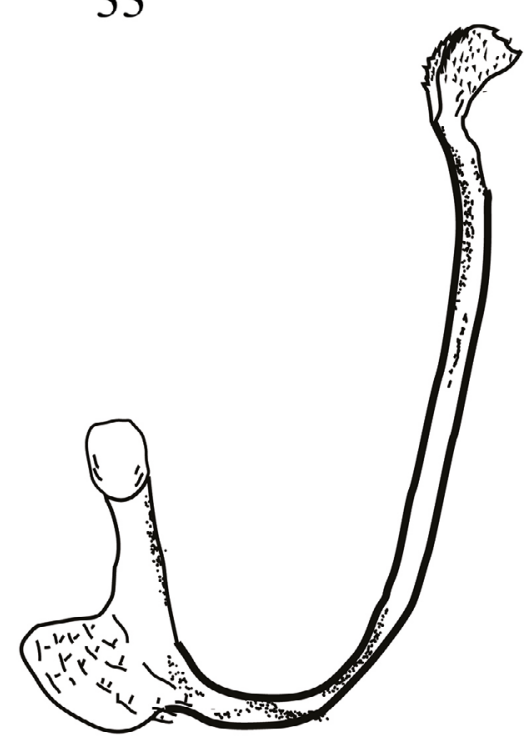

(7-8) dientes primarios, cuatro dientes secundarios y cuatro cerdas marginales (Fig. 31).

Terminalia interna. Hipandrio quitinizado y ovalado sin arco dorsal, la parte anterior membranosa y en la parte posterior con dos proyecciones quitinosas, gonopodio membranoso con una cerda larga (Fig. 32). Edeago quitinizado, largo en forma de "C", el extremo distal ensanchado, borde ventral aserrado, apodema del edeago pequeño y membranoso, rama ventral alargada, paráfisis ovalada (Figs. 30,33,34).
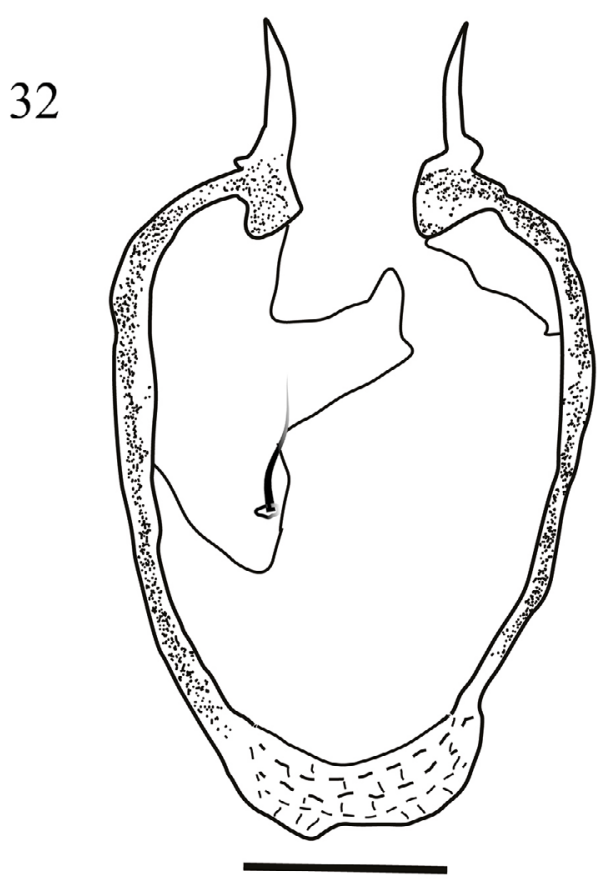

34

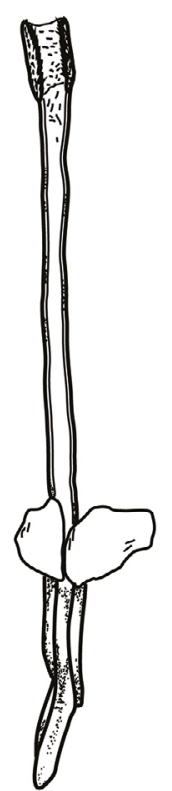

Figs 31-34. Drosophila cartucho sp. nov., holotipo ô: 31, epandrio; 32, hipandrio y gonopodio; 33-34, edeago y paráfisis, vistas lateral y ventral, respectivamente (Escala: $0.1 \mathrm{~mm}$ ). 

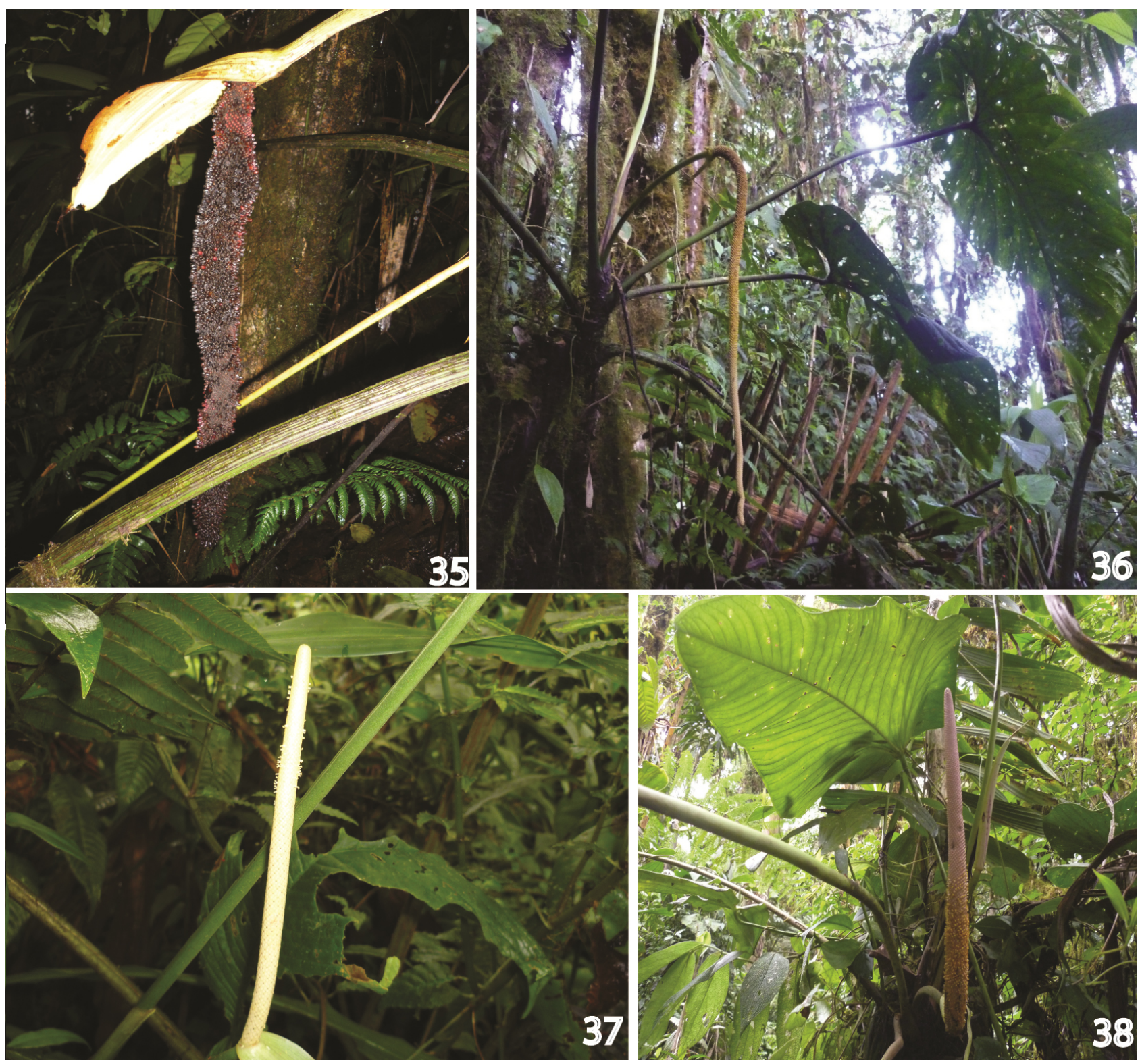

Figs 35-38. Especies de la familia Araceae de la Reserva Río Guajalito relacionadas al género Drosophila: 35, Anthurium sp.; 36, Araceae 1; 37, Araceae 2; 38 , Araceae 3 .

Ecología. Especie capturada en las inflorescencia de varias aráceas entre ellas Anthurium spp. (Fig. 35) y Araceae 3 (Fig. 38).

Etimología. El nombre de la especie hace referencia a "cartucho" nombre popular con el que se conoce a varias especies del género Anthurium.

\section{DISCUSIÓN}

Las cinco especies nuevas descritas fueron capturadas en inflorescencias de Araceae, y poseen características propias a nivel morfológico y ecológico, por lo que no es posible confundirlas con los miembros de los otros grupos de especies relacionados con flores (BRNCIC, 1983; FigUERO et al., 2012).

Según nuestros resultados podríamos agrupar a estas especies en dos grupos. Grupo 1 formado por D. anthurium sp. nov., D. tsachila sp. nov. y D. guajalito sp. nov. y se podría definir como: especies de tamaño mediano (alrededor de $3.5 \mathrm{~mm}$ ), cuerpo amarillento, tórax con 6 hileras de pelos acrosticales entre las dorsocentrales anteriores, cerdas basales divergentes. En D. anthurium sp. nov. y D. tsachila sp. nov. la cerda esternopleural media es casi imperceptible, mientras que en $D$. guajalito sp. nov. no está presente. Abdomen amarillo con pigmentación obscura bien definida, placa anal libre y prominente. La parte dorsal y el lóbulo inferior del epandrio sin cerdas, surestilo sumamente alargado con dientes primarios y secundarios. Hipandrio con arco dorsal. Edeago alargado en forma de "C", con apodema muy reducido en $D$. anthurium sp. nov. y D. guajalito sp. nov., mientras que en $D$. tsachila sp. nov. el apodema es más desarrollado.

Grupo 2 formado por $D$. deloscolorados sp. nov. y D. cartucho sp. nov. se definiría como: especies medianas 
(alrededor de 3,4 mm), cuerpo amarillo, tórax con 6 hileras de pelos acrosticales entre las dorsocentrales anteriores, cerdas basales divergentes, cerda esternopleural media $1 / 5$ de la cerda esternopleural anterior. Abdomen con pigmentación obscura, placa anal libre no prominente. Epandrio con escasas cerdas, lóbulo inferior del epandrio alargado con pocas cerdas, surestilo menos alargados que las del grupo 1, con dientes primarios y secundarios. Hipandrio sin arco dorsal. Edeago en forma de "C" con la parte distal redondeada y el apodema del edeago muy corto en $D$. cartucho sp. nov., y en $D$. deloscolorados más desarrollado.

Los cinco taxones nuevos presentan el edeago alargado como las especies de los otros grupos de drosófilas relacionadas con flores, por ejemplo D. flavopilosa (BRNCIC, 1966, 1983; SANTos \& Vilela, 2005; Vela \& Rafael, 2005); D. bromeliae (VAL \& Marques, 1996; Silva \& Martins, 2004; GRIMALDI, 2016) y D. onychophora (HUNTER, 1979; Vilela \& BÄChLI, 1990; Hunter, 1992; Figuero \& RAFAel, 2011; Figuero et al, 2012).

Existen trabajos que muestran que las aráceas son sitios de reproducción, cría y alimentación de algunas especies de Drosophila (Tsacas \& Chassagnard, 1992; Llangarí \& Rafael, 2017; Vilela \& Prieto, 2018). En este estudio se encontró que $D$. anthurium sp. nov. emergió de la inflorescencia de Anthurium, mientras que $D$. tsachila sp. nov., D. deloscolorados sp. nov., D. guajalito sp. nov. $y$ D. cartucho sp. nov. fueron capturadas sobre la inflorescencia de diferentes especies de aráceas.

Creemos que son necesarios más datos sobre la ecología de estas especies nuevas. Por ejemplo, para conocer detalladamente la relación de las drosófilas con las diferentes fases del desarrollo de las inflorescencias. Sin embargo, podríamos indicar que estas especies tienen preferencia por las aráceas, ya que simultáneamente a este estudio, CABEZAS et al. (2015) realizaron investigaciones sobre el género Drosophila utilizando trampas de banano fermentado con levadura. Estas trampas fueron colocadas aproximadamente a 2 metros de distancia de las plantas muestreadas en el presente trabajo, durante cuatro períodos de colecta. A pesar de ello, CABEZAs et al. (2015) no capturaron ningún individuo de las especies descritas en este estudio.

Futuros estudios sobre drosófilas relacionadas con Araceae permitirán conocer con más detalle aspectos ecológicos de estas especies, y tal vez existe un grupo nuevo antofílico adaptado a esta familia botánica.

Agradecimientos. A la Pontificia Universidad Católica del Ecuador por financiar los proyectos No 13171, H19121 y J13054. Al Dr. Jaime Jaramillo (In memoriam), a la Lic. Elena Grijalva por su colaboración especial y permitirnos trabajar en la Estación Científica Río Guajalito, a la Msc. María Beatriz Cabezas por su ayuda en la fase de campo. Al Ministerio del Ambiente por otorgar los permisos de colección $\mathrm{N}^{\circ}$ 001-10 IC-FAU-DNB/MA y $\mathrm{N}^{\circ}$ 001-11 IC-FAU-DNB/MA.

\section{REFERENCIAS}

Bächli, G.; Vilela, C. R.; Andersson, S. \& Saura, A. 2004. The Drosophilidae (Diptera) of Fennoscandia and Denmark. Fauna Entomologica Scandinava. Vol. 39. Leiden, Brill. 362p.

BRNCIC, D. 1966. Ecological and cytogenetical studies of Drosophila flavopilosa, a Neotropical species living in Cestrum flowers. Evolution 20:16-29.

BRncIC, D. 1983. Ecology of Flower-Breeding Drosophila. In: AsHBURNER, M.; Carson, H. L. \& Thompson, J. N. eds. The genetics and Biology of Drosophila. London, New York, Academy Press, v. 3, p. 333-382.

Cabezas, M. B.; Llangari, L. M. \& Rafael, V. 2015. Descripción de cuatro especies nuevas del subgrupo Drosophila fasciola, grupo repleta (Diptera, Drosophilidae) en dos bosques nublados del Ecuador. Iheringia, Série Zoologia 105(4):383-392.

Carson, H. L. \& OKada, T. 1980. Drosophilidae associated with flowers in Papua New Guinea. I. Colocasia esculenta. Kontyu 48:15-29.

Figuero, M. L. \& Rafael, V. 2011. Dos nuevas especies del grupo Drosophila onychophora (Diptera, Drosophilidae) en los bosques de Polylepis de Papallacta, Pichincha, Ecuador. Iheringia, Série Zoologia 101(4):342-349.

Figuero, M. L.; León, R.; Rafael, V. \& Céspedes, D. 2012. Cuatro nuevas especies del grupo Drosophila onychophora (Diptera, Drosophilidae) en el Parque Arqueológico Rumipamba, Pichincha, Ecuador. Iheringia, Série Zoologia 102(2):212-220.

GRIMALDI, D. 2016. Revision of the Drosophila bromeliae Species Group (Diptera: Drosophilidae): Central American, Caribbean, and Andean Species. American Museum Novitates 3859:1-55.

Heed, W. B \& Wheeler, M. R. 1957. Thirteen new species in the genus Drosophila from the Neotropical region. The University of Texas Publications 5721:17-38.

Hunter, A. S. 1979. New Anthophilic Drosophila of Colombia. Annals of the Entomological Society of America 72:372-383.

Hunter, A. S. 1992. Flower-breeding Drosophila of Bogota, Colombia: new species (Diptera, Drosophilidae). Pan-Pacific Entomologist 68:192-199.

Jaramillo, J. L. \& Grijalva, E. 2010. Flora del bosque nublado de Río Guajalito. 2ed. Quito, Herbario QCA de la Escuela de Biología, Facultad de Ciencias Exactas y Naturales, Pontificia Universidad Católica del Ecuador. 283p.

Llangari, L. M. \& Rafael, V. 2017. A new species of Drosophila (Diptera: Drosophilidae) from the inflorescences of Xanthosoma sagittifolium (Araceae) Revista Ecuatoriana de Medicina y Ciencias Biológicas 38(1):55-60.

PIPKIN, S. 1964. New flower breeding species of Drosophila (Diptera, Drosophilidae). Proceedings of the Entomological Society of Washington 66:217-245.

Pipkin, S.; Rodríguez, R. \& León, J. 1966. Plant host specifity among Flower-Feeding Neotropical Drosophila (Diptera: Drosophilidae) American Naturalist 100:135-156.

RAFAel, V.; Arcos, G. \& Arcos, L. 2000. Ecología y distribución del género Drosophila en Guayllabamba y El Quinche, Provincia de PichinchaEcuador. Revista de la Pontificia Universidad Católica 65:130-155.

Ramos, E. \& Rafael, V. 2018. Two new species in the Drosophila flavopilosa and Drosophila morelia species groups (Diptera: Drosophilidae) in the eastern Andes of Ecuador. Revista Peruana de Biología 25(2):69-74.

Robe, L. J.; De Ré, F. C.; Ludwig, A. \& Loreto, E. L. 2013. The Drosophila flavopilosa species group (Diptera, Drosophilidae): An array of exciting questions. Fly 7:59-69.

Santos, R. \& Vilela, C. R. 2005. Sítios de desenvolvimiento larval de Drosofílideos (Diptera) neotropicais: IV. Flores de Sessea brasiliensis e Cestrum spp. (Solanaceae) coletadas nas plantas e no solo. Revista Brasileira de Entomología 49(4):544-551.

Shi, T.; Toda, M. J.; Takano, K. T.; Yafuso, M.; Suwito, A.; Wong, S. Y.; Shang, S. \& GAO, J. 2019. A review of taxonomy and flowerbreeding ecology of the Colocasiomyia toshiokai species group (Diptera: Drosophilidae), with description of a new species from Indonesia. European Journal of Entomology 116:341-361. 
Silva, A. \& Martins, M. 2004. A new anthophilic species of Drosophila Fallén belonging to the bromeliae group of species (Diptera, Drosophilidae). Revista Brasileira de Zoologia 21:435-437.

Sturtevant, A. H. 1921. Genetic studies on Drosophila simulans. II. Genetics 6:43-64

Tsacas, L. \& Chassagnard, M. T. 1992. Les relations Araceae Drosophilidae. Drosophila araceae une espèce anthophile associèe Xanthosoma robustum au Mexique (Diptera: Drosophilidae). Annales de la Société Entomologique de France 28(4):421-439.

Val, F. C. \& Marques, M. D. 1996. Drosophilidae (Diptera) from the Pantanal of Mato Grosso (Brazil), with the description of a new species belonging to the bromeliae group of the genus Drosophila. Papéis Avulsos de Zoologia 39:223-230.

Vargas, J. H.; Consiglio, T.; Jørgensen, P. M. \& Croat, T. B. 2004. Modelling distribution patterns in a species-rich plant genus, Anthurium (Araceae), in Ecuador. Diversity and Distribution 10:211-216.

Vaz, S. C.; Vilela, C. R.; Krsticevic, F. J. \& Carvalho, A. B. 2014. Developmental Sites of Neotropical Drosophilidade (Diptera): V. Inflorescences of Calathea cylindrical and Calathea monophylla
(Zingiberales: Marantaceae). Annals of the Entomological Society of America 107(3):607-620.

Vela, D. \& Rafael, V. 2005. Catorce nuevas especies del género Drosophila (Diptera, Drosophilidae) en el Bosque húmedo montano del Volcán Pasochoa, Pichincha, Ecuador. Revista Ecuatoriana de Medicina y Ciencias Biológicas 27(1,2):27-41.

Vilela, C. R. \& BäChli, G. 1990. Taxonomic studies on Neotropical species of seven genera of Drosophilidae (Diptera). Mitteilungen der Schweizerischen Entomologischen Gesellschaft Bulletin de la Société Entomologique Suisse 63:1-332.

VILELA, C. R. \& BÄCHLI, G. 2004. Revision of the Drosophila macroptera and $D$. rubrifrons species group, with description of a new Neotropical group (Diptera, Drosophilidae). Mitteilungen der Schweizerischen Entomologischen Gesellschaft Bulletin de la Société Entomologique Suisse 77:1-68.

Vilela, C. R. \& Prieto, D. 2018. A new Costa Rican species of Drosophila visiting inflorescences of the hemi-epiphyctic climber Monstera lentii (Araceae). Revista Brasileira de Entomologia 62:225-231. 